\title{
¿Blanco, negro o escala de grises? Determinación de la contribución relativa del nicho ecológico y la teoría neutral en los ensambles de especies
}

\author{
Juan Alberti ${ }^{\varpi}$; Pedro Daleo \& Oscar Iribarne \\ Instituto de Investigaciones Marinas y Costeras (IIMyC) - FCEyN, Universidad Nacional de Mar del Plata, Consejo Nacional \\ de Investigaciones Científicas y Técnicas (CONICET).
}

\begin{abstract}
Resumen. Según la teoría del nicho ecológico, la composición de especies en un lugar dado debería estar determinada por la conjunción de los factores bióticos y abióticos que allí actúan. En cambio, la teoría neutral propone que las especies son equivalentes y que la composición de ensambles locales está dada, entonces, por procesos de colonización y extinción independientes de las especies. Hoy en día se sabe que es difícil encontrar en la naturaleza ensambles puramente neutrales o puramente basados en el nicho ecológico, sino que lo que priman son ensambles intermedios. Los ensambles con una impronta fuerte del nicho ecológico están muy influidos por las interacciones interespecíficas o por los forzantes ambientales, mientras que los ensambles neutrales están más influidos por la deriva ecológica o por las dinámicas de extinción y colonización en base a las abundancias de las especies. El concepto de beta diversidad se tornó especialmente popular durante la última década y ha sido utilizado con diferentes finalidades en una gran variedad de ambientes. En particular, ganó mucha relevancia como herramienta para estimar la importancia relativa del nicho ecológico en los ensambles de especies. Esto se puede evaluar de forma sencilla con el uso de modelos neutrales ad hoc. En un ensamble puramente neutral, la variabilidad en la composición del ensamble entre muestras no debería ser diferente a la esperada como producto de una selección de especies al azar del total regional de especies (diversidad gama) para cada muestra (diversidad alfa). Cuanto más se aleje la variabilidad a la esperable por azar, mayor será la importancia relativa del nicho ecológico. Recientemente, hubo un gran avance en entender qué tipos de factores promueven uno u otro ensamble. Sin embargo, al ser un campo relativamente nuevo queda aún mucho por saber (e.g., interacción de factores, relación tiempoespacio, diferencias entre niveles tróficos). En este trabajo revisamos los alcances y la metodología de una temática que está viviendo un desarrollo marcado dentro de la ecología de comunidades, y discutimos algunos de los aspectos relevantes y relacionados que, por el momento, no fueron estudiados.
\end{abstract}

[Palabras clave: patrones de diversidad, modelo nulo]

Aвstract. Using beta-diversity to differentiate neutral from niche community assembly. According to the niche theory, species assemblies should reflect biotic and abiotic local conditions. In turn, the neutral theory of biodiversity stands that species are equivalent and that local species assembly is the product of colonization and extinction rates. After some years of debate, nowadays, ecologists agree that purely niche or neutral assemblies rarely occur in nature and, in turn, that the rule are assemblies somewhere in the middle. A niche assembly is the product of interspecific interactions or environmental forcing, while a neutral assembly is derived from intrinsic population dynamics (e.g., colonization and extinction rates). The beta-diversity concept (it has various definitions, but all of them share the idea of a link between local [alpha] and regional [gamma] diversity, and, thus, refers to the heterogeneity in species composition between places or times) has become particularly popular during the last decade. It is used to analyze a great variety of systems in different ways. In particular, it gained attention as a tool to differentiate niche- vs neutral-based species assembly. This could be easily addressed using ad hoc neutral models. A pure neutral assembly should exhibit a variability in species composition similar to that expected by randomly selecting species from the regional pool (gamma diversity) for each sample (alpha diversity). The more deviant from the variability expected by chance, the more niche-based assembly. We have greatly expanded our knowledge of the factors that promote one or the other type of assembly. However, there is still much more to elucidate, like how neutral vs. niche assembly is influenced by the interaction between factors, spatio-temporal relationships or if there are scale-dependent variations. In this review, we analyze the potential uses and methodologies associated with this emerging topic in community ecology and discuss some related and interesting scientific questions that remain unanswered.

[Keywords: ecological niche, neutral theory, diversity patterns, null model] 


\section{INTRODUCCIÓN}

Uno de los objetivos centrales de la ecología, desde su aparición como disciplina científica, es conocer los factores que determinan la diversidad de las comunidades (Hutchinson 1959). Desde muy temprano, el foco estuvo centrado en la influencia de las condiciones abióticas como reguladoras casi exclusivas de la composición de especies y de sus cambios temporales en un determinado lugar (Clements 1916). Luego, la presencia de diferentes especies en las comunidades locales pasó a ser interpretada como la consecuencia de una acumulación estocástica e histórica de especies producida por dinámicas de colonización y extinción (MacArthur and Wilson 1967). Poco después, la visión de que la diversidad podía ser explicada por la competencia, los consumidores y la partición de nichos concentró mucha atención (Paine 1966; MacArthur 1972; Menge and Sutherland 1976; McNaughton 1983). Más recientemente, se incorporaron los efectos de las interacciones positivas (en especial la facilitación) a la teoría del nicho ecológico (Stachowicz 2001; Bruno et al. 2003). Desde entonces existió cierto debate sobre si la diversidad en un determinado lugar era explicada por procesos relacionados con el nicho ecológico o por procesos neutrales. Este debate recrudeció después de que Stephen Hubbell publicó su libro sobre la teoría neutral, donde postula que las especies son equivalentes y que la composición de los ensambles está dada por dinámicas de colonización y extinción independientes de sus identidades (Hubbell 2001). De acuerdo con el nicho ecológico, la composición de una comunidad está determinada por interacciones biológicas y condiciones abióticas que actúan como fuerzas determinísticas que definen ensambles con miembros limitados y predecibles (Hutchinson 1957). Por el contrario, la teoría neutral postula que los ensambles de especies están principalmente influenciados por procesos estocásticos (e.g., colonización y extinción, muertes aleatorias), que llevan a cambios en las abundancias relativas independientes de las condiciones ambientales y que provocan ensambles más impredecibles (Hubbell 2001). Hoy en día existe un consenso amplio en que estos dos tipos de procesos coexisten (Leibold and McPeek 2006; Adler et al. 2007). El desafío actual, entonces, no es determinar cuál de las teorías es la correcta, sino conocer los factores que regulan su importancia relativa (Chase 2007; Chase and Myers 2011).
Una forma de explorar la importancia relativa del nicho ecológico y de los procesos neutrales en los ensambles de especies es analizando la beta diversidad. El concepto de beta diversidad fue propuesto por Whittaker (1960) hace más de medio siglo, pero recién en la última década ganó un interés marcado entre los ecólogos. A lo largo del tiempo, este concepto fue reinterpretado y reformulado muchas veces, pero, en esencia, todas las definiciones comparten la idea de que es un nexo entre la diversidad a nivel local (alfa) y la diversidad a nivel regional (gama) (Tuomisto 2010). Tan importante se volvió el término que en la actualidad está en el centro de la ecología de comunidades (Anderson et al. 2011). En las diferentes definiciones de beta diversidad, sus relaciones con la diversidad alfa y gama van desde las estrictamente matemáticas (e.g., aditiva o multiplicativa) a aproximaciones multivariadas, en las que se toma en cuenta la disimilitud entre parcelas (Anderson et al. 2006; Tuomisto 2010). El estudio de la diversidad beta puede ser muy útil para responder numerosas preguntas ecológicas medianteíndices y procedimientos estadísticos pertinentes a esas preguntas (Anderson et al. 2011). De hecho, existe una variedad muy notoria de ambientes y de sistemas en los que se la utilizó para responder una gama amplia de preguntas que van desde mecanismos de ensamble comunitario en bosques tropicales y templados (Myers et al. 2013) hasta variabilidad espacial y temporal en ensambles de aves (Stegen et al. 2013a), pasando por la homogeneización de comunidades de plantas de bosque producto de la agricultura (Vellend et al. 2007), los mecanismos de sucesión en bacterias (Dini-Andreote et al. 2015) y los patrones de cambio espacial y temporal en la composición de especies de diferentes sistemas y posiciones en las tramas tróficas (Soininen et al. 2007; Korhonen et al. 2010).

Cualquiera sea el caso, otra forma de ver a la diversidad beta es como un término que refiere a la heterogeneidad en la composición entre lugares o tiempos (Figura 1). Cuanto más diferentes sean los ensambles de especies entre lugares o tiempos (a cada combinación de lugar y tiempo le corresponde una diversidad alfa), mayor será la diversidad beta. Uniendo esta idea con la teoría de los grandes números surgió una de las utilizaciones del concepto de diversidad beta que ganó gran atención durante los últimos años: una herramienta para evaluar la importancia relativa del nicho ecológico y de los procesos neutrales en los 


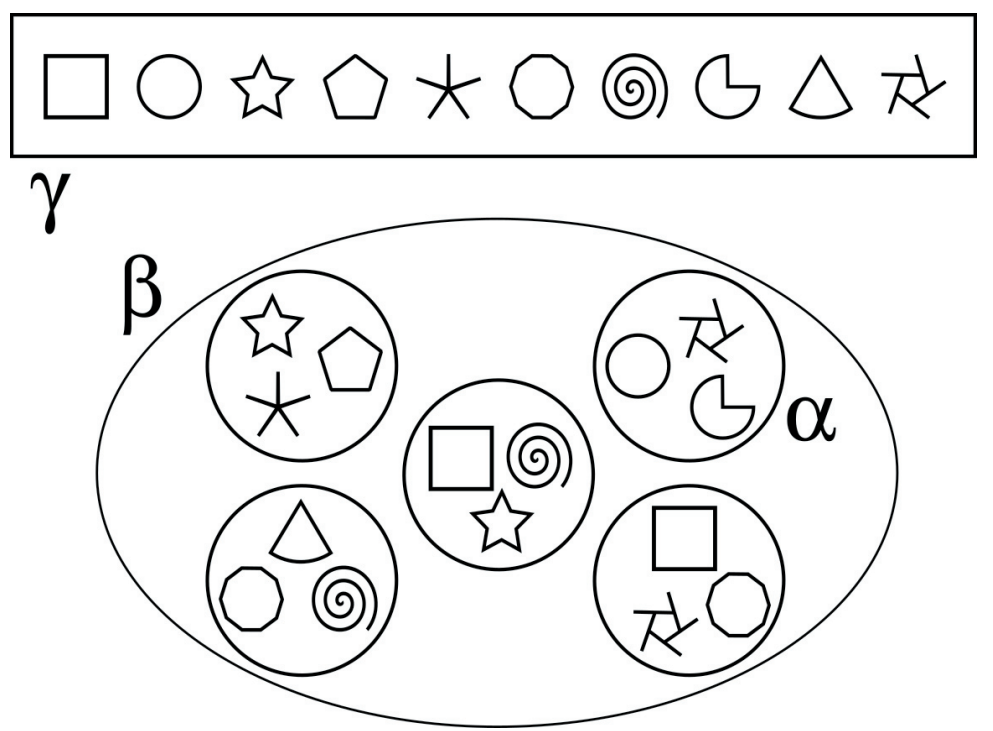

Figura 1. Esquema que representa las relaciones de las distintas escalas de diversidad. La diversidad gama está conformada por la composición de especies a nivel regional (rectángulo superior). Cada uno de los símbolos dentro de este rectángulo representa a una especie diferente. La diversidad alfa está conformada por la composición a escala local (círculos con tres especies dentro del óvalo) y la diversidad beta está dada por las diferencias entre composiciones a escala local (óvalo). La diversidad alfa refiere a la composición de especies en un tiempo y lugar dado. Por lo tanto, en el esquema, los diferentes círculos pueden representar diferentes lugares o tiempos.

Figure 1. Schematic representation of the relationships of different scales of diversity. Gamma diversity is represented by the upper rectangle which includes species composition at the regional scale. Alpha diversity is represented by circles arbitrary containing three species at the local scale, and beta diversity is given by the differences in composition between local scales. Alpha diversity represents the species composition at a given time and place. Thus, different circles could represent different times or locations.

ensambles de especies (Chase et al. 2011; Tucker et al. 2016).

La utilidad de la diversidad beta para determinar la contribución relativa de la teoría de nichos y la teoría neutral radica en que los patrones esperables de variación en la composición y la abundancia difieren entre estas dos teorías. Los procesos relacionados con el concepto de nicho ecológico incluyen la selección impuesta por el ambiente (Kraft et al. 2015) y las interacciones de sinergia y antagonismo entre las especies. En términos generales, estos procesos llevan a comunidades convergentes, con muy baja variación en la composición de especies entre sitios (diversidad beta baja) cuando las condiciones ambientales son similares (Chase et al. 2009; Chase and Myers 2011) (Figura 2). Por otra parte, los procesos neutrales están relacionados con la teoría neutral (Hubbell 2001), e incluyen las dinámicas de colonización y de extinción, así como la deriva ecológica, por lo que suelen llevar a comunidades divergentes, con una variación elevada en la composición de especies entre sitios (diversidad beta alta), incluso cuando las condiciones ambientales son similares (Chase and Myers 2011) (Figura 2). En tiempos ecológicos, el ensamble de especies en una comunidad local también está influenciado por la dispersión (Vellend 2010). Si bien la dispersión podría actuar tanto como proceso neutral como ligado al nicho ecológico (Vellend 2010; Vellend et al. 2014), un meta-análisis reciente sugiere que su influencia es principalmente mediante efectos de muestreo (neutrales) (Catano et al. 2017). Más allá de esto, nos vamos a centrar en cómo distinguir la importancia relativa de procesos neutrales y relacionados al nicho ecológico en los ensambles de especies para poder estudiar si existen determinadas condiciones que afecten a dicha importancia relativa. Para poder discernir en qué punto de este gradiente se encuentra un ensamble dado se utilizan modelos neutrales ad hoc (Chase et al. 2011; ver Gotelli and McGill 2006 para una discusión de las diferencias entre modelos nulos y neutrales). En este punto vale aclarar que hay un gran número de trabajos en los que se siguieron estas aproximaciones, pero se discute la contribución relativa de procesos estocásticos y determinísticos en lugar de la de ensambles neutrales y relacionados al nicho ecológico. Nosotros consideramos que esta segunda forma de interpretar los resultados es la correcta (Tucker et al. 2016), por lo que en este trabajo seguiremos esta terminología. 


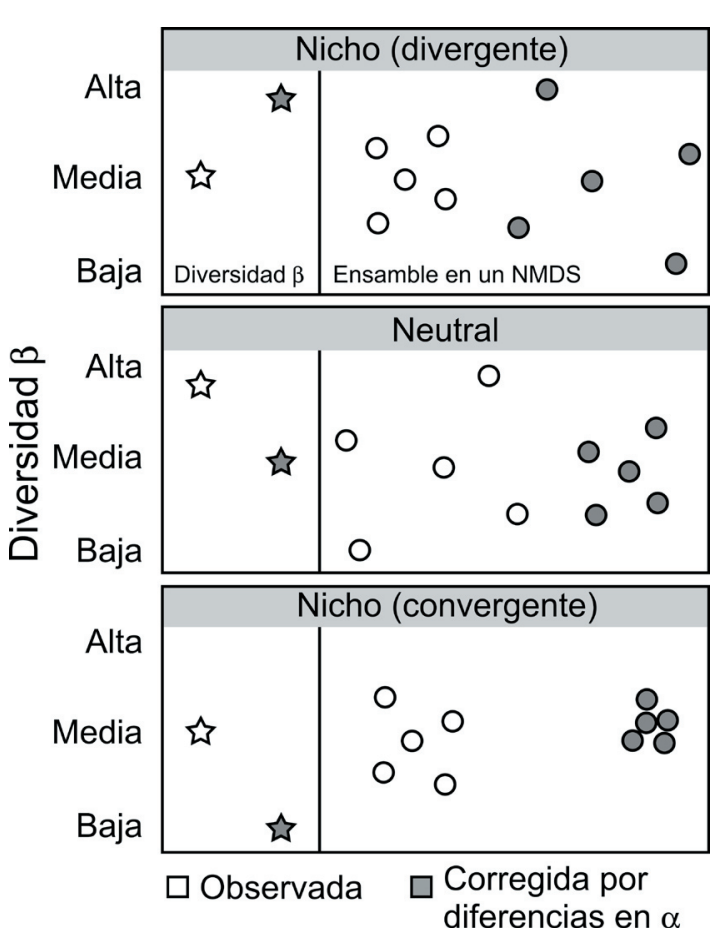

Figura 2. Escenarios hipotéticos que muestran cualitativamente la relación entre la diversidad beta y los posibles mecanismos de ensamble comunitario. A partir de la diversidad beta observada (símbolos blancos) y de sus valores luego de ser corregida por la diversidad alfa observada (símbolos grises) se puede determinar la importancia relativa de procesos neutrales y determinísticos en los ensambles de las comunidades. Los ensambles determinísticos son aquellos con mayor (panel superior) o menor variación (panel inferior) de la esperada por azar (panel intermedio). Cuanto mayor sea la desviación con respecto al modelo neutral (positiva para comunidades divergentes o negativa para convergentes), mayor será la importancia de procesos determinísticos en el ensamble de la comunidad. Los ensambles convergentes tienen composiciones muy similares y, por ende, se manifiestan con puntos más agrupados en un gráfico de NMDS. Los ensambles puramente neutrales presentan niveles intermedios de dispersión entre los puntos, mientras que las máximas dispersiones se observan en ensambles divergentes (e.g., con condiciones ambientales heterogéneas se pueden expresar comunidades diferentes para cada condición).

Figure 2. Hypothetical scenarios to show the relationship between beta diversity and community assembly mechanisms. The relative importance of neutral and deterministic community assembly can be determined with the observed beta diversity (white symbols) and after correcting it for observed alpha diversity (gray symbols). Deterministic assemblies are those with higher (top panel) or lower (bottom panel) variability than expected by chance (intermediate panel). The higher the deviation from the neutral model (either positive for divergent or negative for convergent communities) the higher the importance of deterministic community assembly. Deterministic convergent communities are highly similar in species composition than expected by chance and thus, in a NMDS plot, they show highly clustered points. A purely neutral assembly shows intermediately scattered points, while maximum dispersion is observed in deterministically divergent communities (e.g., in heterogeneous abiotic and/or biotic conditions, environmental filtering can lead to a different community for each environmental condition).

\section{¿QUÉ SABEMOS?}

Sin dudas, se ha avanzado mucho en los últimos años en entender qué factores regulan la importancia relativa de los diferentes tipos de ensambles de especies. Por ejemplo, se demostró que aumentar la disponibilidad de nutrientes puede llevar a ensambles más neutrales (Chase 2010; Steiner 2014) mediante efectos de prioridad (las especies que primero arriban a una parcela determinan la composición final del ensamble) (Fukami 2015). Esto ocurre principalmente porque el exceso de nutrientes le permite aumentar las tasas de crecimiento a las primeras especies en colonizar la parcela, lo que disminuye las posibilidades de un asentamiento exitoso de las especies que llegarán después, más allá de sus jerarquías competitivas. Los disturbios y la dispersión también son factores que promueven la importancia relativa de los ensambles algo más neutrales. Estos factores tienden a disminuir las dominancias locales, lo que permite que se establezcan numerosas especies subordinadas ("muestreadas" al azar del total regional de especies) (Catano et al. 2017). Por otra parte, entre los factores que promueven ensambles basados en el nicho ecológico se encuentran los consumidores (depredadores: Chase et al. 2009; granívoros: Germain et al. 2013; herbívoros: Alberti et al. 2017), que actúan como filtros que eliminan de los ensambles locales a las especies más susceptibles a ser consumidas, o promueven la dominancia de aquellas más tolerantes a los consumidores. El estrés abiótico es otro factor que aumenta la importancia relativa del nicho ecológico y que lleva a la convergencia de los ensambles locales a partir de excluir a aquellas especies que no toleran las condiciones estresantes (sequía: Chase 2007; salinidad: Guo et al. 2014). Por lógica, si cada factor que promueve la importancia relativa del nicho ecológico genera un ensamble propio y convergente, parcelas sujetas a diferentes factores (o a condiciones ambientales heterogéneas) mostrarían una mayor diversidad beta (Anderson et al. 2006). En este caso, el nicho ecológico promovería ensambles divergentes (más divergentes de lo esperado por azar), como se ha observado en aves a lo largo de todo Estados Unidos (Stegen et al. 2013a) o en ensambles de oribátidos en bosques y pastizales de Italia (Caruso et al. 2012b).

Estas últimas evidencias podrían llevar a pensar que aquellos factores asociados 
al nicho ecológico necesariamente llevan a ensambles menos neutrales, en términos relativos. Sin embargo, esto no siempre es así, y vamos a ilustrar por qué. Hace más de medio siglo que se demostró la importancia de las jerarquías competitivas en la supervivencia y la distribución de los organismos (Connell 1961). Un ejemplo paradigmático de los efectos de la competencia se da en pastizales, en los que la competencia por la luz entre plantas puede tener un efecto marcado en la riqueza vegetal. En ese contexto, aquellos factores que aumentan la competencia por luz llevan a pérdidas notables en la riqueza de especies subordinadas, como sucede con aumentos en la disponibilidad de nitrógeno que llevan a un mayor crecimiento de los pastos altos dominantes que sobrecompiten a los subordinados, reduciendo así la diversidad (Hautier et al. 2009). En el mismo sentido, cuando los herbívoros, al consumir a las especies altas dominantes, son capaces de aumentar la llegada de luz a los estratos más bajos de la vegetación, también aumentan la diversidad vegetal (Borer et al. 2014). Si bien todos estos procesos están claramente relacionados con elnichoecológico, el resultado de sus efectos no siempre son ensambles convergentes basados en el nicho. Cuando el gremio de los pastos altos dominantes se compone de varias especies que se excluyen entre ellas, o cuando no todas las especies subordinadas son eliminadas a escala local, sino sólo algunas de ellas (y de forma aleatoria entre las diferentes parcelas), el resultado de la competencia (relacionada con el nicho en el sentido de que los pastos altos dominantes excluyen competitivamente a pastos cortos) son ensambles mayormente neutrales en los que la identidad de las especies dominantes y/o subordinadas varía entre parcelas por procesos neutrales (Segre et al. 2014).

El balance entre ensambles neutrales y basados en el nicho ecológico no sólo puede cambiar con diferentes factores, sino que tampoco es uniforme entre los diferentes seres vivos. Las comunidades microbianas, por ejemplo, presentan ensambles con una impronta neutral muy marcada, en especial a pequeña escala (Caruso et al. 2011; Zhou et al. 2013; Dini-Andreote et al. 2015; Powell et al. 2015). Esto estaría dado por las tasas de dispersión y de inestabilidad ambiental muy elevadas a esa escala (Dini-Andreote et al. 2015). De hecho, al aumentar el aislamiento de las comunidades, o al contemplar los efectos de la inestabilidad ambiental, disminuye esa impronta neutral (Powell et al. 2015). Algo similar ocurre al aumentar la escala espacial, o al considerar simultáneamente varios grupos funcionales, porque esto implica barreras muy fuertes a la dispersión o diferencias muy marcadas en el nicho ecológico (Caruso et al. 2011). La capacidad dispersiva, entonces, parece ser una de las principales causas de las diferencias en el tipo de ensamble entre los distintos grupos de seres vivos. En comparación, los hongos presentan ensambles más relacionados con el nicho ecológico que las bacterias, y esto fue asociado a los tamaños de las esporas y al alcance de su dispersión (Brown and Jumpponen 2014; Schmidt et al. 2014).

En términos generales, aquellos factores que promueven la dispersión o la homogeneización y el mejoramiento de las condiciones ambientales tienden a aumentar la importancia relativa de ensambles neutrales. Por otro lado, aquellos factores que imponen condiciones ambientales (incluyendo factores bióticos y abióticos) más estresantes suelen aumentar la importancia relativa del nicho ecológico y conducen a ensambles de especies más convergentes. La importancia relativa del nicho ecológico también aumenta cuando se estudian ambientes con condiciones muy heterogéneas, lo que lleva a ensambles divergentes. Esto es así porque para cada condición se generan ensambles propios diferentes del resto. En este contexto, lo mismo debería suceder con cualquier factor que promueva la heterogeneidad espacial o temporal.

\section{¿Cómo lo SABemos?}

Existen muchas aproximaciones para evaluar la importancia de diferentes características y procesos de los ensambles, como, por ejemplo, la capacidad de dispersión que ya discutimos, pero también la distancia genética, la estructuración espacial o la deriva ecológica. Cuanto mayor sea el grado de detalle que se pretenda para poder discernir su efecto, mayor será el grado de complejidad que tendrán los análisis (Etienne 2007, 2009; Caruso et al. 2012a; Stegen et al. 2013b). Por su simplicidad y utilidad, en este trabajo nos vamos a centrar en lo más general, que son aquellos análisis que permiten discriminar la contribución relativa de los ensambles neutrales y de aquellos basados en el nicho ecológico, sin discriminar entre sus posibles componentes. 
El principal problema de inferir tipos de ensambles a partir de patrones de diversidad beta es que como las tres escalas de diversidad (alfa, beta y gama) están relacionadas entre sí, las diferencias en diversidad beta pueden ser la resultante de procesos que realmente modifiquen las reglas de ensamble, o bien de procesos que sólo conduzcan a cambios en diversidad alfa (Chase et al. 2011), gama (Kraft et al. 2011) o en ambas (Sabatini et al., en prensa). Si se desea inferir que procesos están detrás de los cambios en los patrones de diversidad beta entre ensambles con diferente diversidad alfa, es clave tener en cuenta estas diferencias, porque aquellos sitios con mayor diversidad alfa tendrán matemáticamente una menor diversidad beta, sin que ello implique cambios en el tipo de ensamble. Imaginemos un campo al que se le aplican dos tratamientos (con varias repeticiones cada uno): con y sin agregado de fertilizantes. Sin dudas, los dos tratamientos tendrán la misma diversidad gama (la diversidad observada en el campo entero), pero es probable que el tratamiento con fertilizantes tenga una diversidad alfa menor (Harpole and Tilman 2007; Clark and Tilman 2008). Si la composición en cada repetición fuera la resultante de un muestreo al azar de la diversidad gama, sería esperable que el tratamiento con menor diversidad alfa tenga mayor diversidad beta (cuanto menor representada esté la diversidad gama en cada una de las repeticiones, mayores son las posibilidades de que aumente la divergencia en composición entre las repeticiones). La misma lógica se aplica para diferencias en diversidad gama. Por ejemplo, cuanto mayor sea la diversidad gama, y para una diversidad alfa dada, aumenta la probabilidad de obtener una mayor diversidad beta simplemente por efectos de muestreo. Cuántas más opciones haya para elegir, si se ubican a esas opciones al azar en diferentes parcelas, mayor será la probabilidad que las parcelas difieran en composición. Por todo esto es que se realizan modelos neutrales ad hoc para establecer si las diferencias observadas en diversidad beta son esperables por azar (ensambles neutrales) o no (ensambles relacionados con el nicho ecológico, ya sea convergentes o divergentes).

Entre los modelos neutrales están aquellos que contemplan exclusivamente la identidad de las especies (presencia/ausencia) sin tener en cuenta sus abundancias (Chase et al. 2011), y aquellos que sí contemplan las abundancias (Kraft et al. 2011; Stegen et al. $2013 b)$. En general, los modelos neutrales que contemplan abundancias tienen en cuenta los patrones de distribución de abundancia de las especies (Mori et al. 2015). Es decir, la probabilidad de abundancia de las diferentes especies no va a ser igual entre ellas, sino que las especies que efectivamente sean más abundantes tendrán una mayor probabilidad de ser las más abundantes en las sucesivas simulaciones. Esto fue criticado por algunos investigadores, quienes alegan que un modelo neutral no debería incluir ninguna parte de la diversidad beta que esté determinada por mecanismos de ensamble comunitario (o sea, que todas las especies deberían tener la misma probabilidad de abundancia) (Qian et al. 2013). Sin embargo, esta crítica resulta poco realista en términos ecológicos, ya que, siguiendo esa lógica, las especies típicamente subordinadas podrían ser dominantes en las simulaciones (y viceversa), por lo que no sólo se recomienda la incorporación de la abundancia de las especies sino de sus patrones de distribución (Ulrich and Gotelli 2010; Mori et al. 2015; Tucker et al. 2016). En otras palabras, un modelo neutral no debería tratar a todas las especies con idéntica probabilidad de ocurrencia y abundancia, sino que se debe tener en cuenta que tan comúnmente aparecen y en qué abundancias.

La idea básica que subyace a estos modelos es generar al azar un número grande de ensambles de especies para luego comparar la diversidad beta observada con las generadas aleatoriamente. Sin embargo, estos ensambles generados al azar tienen restricciones que son pertinentes a la pregunta que se quiera evaluar (Mori et al. 2015), pero que, en los casos más comunes (teniendo en cuenta las abundancias de las especies), implican que en estos ensambles: 1) el número de especies en una parcela dada sea el mismo que el número observado para esa parcela; 2) la probabilidad de cada especie de ser asignada a una parcela dependa de su frecuencia de ocurrencia observada entre todas las parcelas; 3) la abundancia total en una parcela dada sea la misma que la abundancia observada en esa parcela, y 4) la probabilidad de abundancia de cada especie dependa de su patrón de distribución de abundancia observado entre todas las parcelas. Si bien la implementación de estos modelos neutrales puede parecer muy compleja para quienes no estén familiarizados, la realidad es que se encuentran publicados varios códigos de $\mathrm{R}$ de diferentes modelos (Chase et al. 2011; Kraft et al. 2011; Stegen et al. 2013a; Sabatini et al. en prensa). A modo de 
ejemplo, en el apéndice incluimos una rutina de $R$ que ilustra los tres tipos de escenarios (factores que promueven ensambles neutrales y ensambles convergentes o divergentes basados en el nicho ecológico), así como la forma de evaluarlos.

\section{CONCLUSIONES}

Si bien se ha avanzado mucho en este campo, sigue siendo mucho lo que queda por responder. 1) El caso que quizás sea más notorio es cómo la interacción entre factores regula el balance entre los dos tipos de ensambles. Se puede suponer que debería aplicarse la misma lógica que para los factores individuales, pero existe muy poca evidencia que lo confirme. Suponemos que en los casos que los diferentes factores evaluados promuevan un mismo tipo de ensamble, la acción conjunta de esos factores debería ir en la misma línea. 2) Otro punto muy poco explorado es el de las sucesiones. DiniAndreote et al. (2015) propusieron un modelo conceptual en el que los primeros ensambles luego de un disturbio serían mayormente neutrales, mientras que la importancia del nicho ecológico iría aumentando con el paso del tiempo, ya sea llevando a ensambles convergentes o divergentes según el contexto. Hasta el momento, este modelo ha recibido poco apoyo empírico y sólo se lo evaluó en forma experimental en ensambles microbianos. Más allá de que es un modelo bien fundamentado en su aspecto teórico, sería deseable avaluar su desempeño en una variedad de sistemas naturales y con los diferentes modelos de sucesión (de facilitación, de inhibición y de tolerancia). 3) Tampoco se ha explorado si los factores que promueven la importancia relativa de uno u otro tipo de ensamble en un determinado nivel trófico se transmiten a otros niveles tróficos.

Para terminar, queda claro que se avanzó notablemente y que todavía resta mucho por entender sobre cómo se modula la importancia relativa de ensambles estocásticos y vinculados al nicho ecológico. Existen numerosas herramientas informáticas para explorar estas cuestiones, pero es importante entender la lógica de lo que se está evaluando, así como el mecanismo subyacente. En primer lugar, es fundamental tener claro qué es lo que se quiere evaluar para elegir qué modelo aplicar. En segundo lugar, también es necesario aplicar estas herramientas a consciencia y no ciegamente, ya que las conclusiones son muy sensibles a las decisiones que se tomen. Una definición inapropiada de diversidad gama, una identificación incorrecta de las especies o un cálculo equivocado de sus abundancias relativas pueden llevar a interpretaciones erróneas.

Agradecimientos. Agradecemos al Dr. James Stegen por brindarnos el código de $\mathrm{R}$ para la realización de modelos neutrales que contemplen las abundancias de las especies. También agradecemos a Martín Núñez y Mariano A. Rodríguez Cabal por sus comentarios que mejoraron la versión final de este trabajo. Este trabajo fue financiado por CONICET, por FONCyT y por la Universidad Nacional de Mar del Plata.

\section{REFERENCIAS}

Adler, P. B., J. HilleRisLambers, and J. M. Levine. 2007. A niche for neutrality. Ecology Letters 10:95-104.

Anderson, M. J., T. O. Crist, J. M. Chase, M. Vellend, B. D. Inouye, A. L. Freestone, N. J. Sanders, H. V. Cornell, L. S. Comita, K. F. Davies, S. P. Harrison, N. J. B. Kraft, J. C. Stegen, and N. G. Swenson. 2011. Navigating the multiple meanings of beta diversity: a roadmap for the practicing ecologist. Ecology Letters 14:19-28.

Anderson, M. J., K. E. Ellingsen, and B. H. McArdle. 2006. Multivariate dispersion as a measure of beta diversity. Ecology Letters 9:683-693.

Borer, E. T., E. W. Seabloom, D. S. Gruner, W. S. Harpole, H. Hillebrand, E. M. Lind, P. B. Adler, J. Alberti, T. M. Anderson, J. D. Bakker, L. Biederman, D. Blumenthal, et al. 2014. Herbivores and nutrients control grassland plant diversity via light limitation. Nature 508:517-520.

Brown, S. P., and A. Jumpponen. 2014. Contrasting primary successional trajectories of fungi and bacteria in retreating glacier soils. Molecular Ecology 23:481-497.

Bruno, J. F., J. J. Stachowicz, and M. D. Bertness. 2003. Inclusion of facilitation into ecological theory. Trends in Ecology and Evolution 18:119-125.

Caruso, T., Y. Chan, D. C. Lacap, M. C. Y. Lau, C. P. McKay, and S. B. Pointing. 2011. Stochastic and deterministic processes interact in the assembly of desert microbial communities on a global scale. The ISME Journal 5:14061413.

Caruso, T., J. R. Powell, and M. C. Rillig. 2012a. Compositional divergence and convergence in local communities and spatially structured landscapes. PLOS ONE 7:e35942.

Caruso, T., M. Taormina, and M. Migliorini. 2012b. Relative role of deterministic and stochastic determinants of soil animal community: a spatially explicit analysis of oribatid mites. Journal of Animal Ecology 81:214-221. 
Catano, C. P., T. L. Dickson, and J. A. Myers. 2017. Dispersal and neutral sampling mediate contingent effects of disturbance on plant beta-diversity: a meta-analysis. Ecology Letters 20:347-356.

Chase, J. M. 2007. Drought mediates the importance of stochastic community assembly. Proceedings of the National Academy of Sciences of the United States of America 104:17430-17434.

Chase, J. M. 2010. Stochastic community assembly causes higher biodiversity in more productive environments. Science 328:1388-1391.

Chase, J. M., E. G. Biro, W. A. Ryberg, and K. G. Smith. 2009. Predators temper the relative importance of stochastic processes in the assembly of prey metacommunities. Ecology Letters 12:1210-1218.

Chase, J. M., N. J. B. Kraft, K. G. Smith, M. Vellend, and B. D. Inouye. 2011. Using null models to disentangle variation in community dissimilarity from variation in $\alpha$-diversity. Ecosphere 2:art24.

Chase, J. M., and J. A. Myers. 2011. Disentangling the importance of ecological niches from stochastic processes across scales. Philosophical Transactions of the Royal Society B: Biological Sciences 366:2351-2363.

Clark, C. M., and D. Tilman. 2008. Loss of plant species after chronic low-level nitrogen deposition to prairie grasslands. Nature 451:712-715.

Clements, F. E. 1916. Plant succession: an analysis of the development of vegetation. Carnegie Institution of Washington.

Connell, J. H. 1961. The influence of interspecific competition and other factors on the distribution of the barnacle Chthamalus stellatus. Ecology 42:710-723.

Dini-Andreote, F., J. C. Stegen, J. D. van Elsas, and J. F. Salles. 2015. Disentangling mechanisms that mediate the balance between stochastic and deterministic processes in microbial succession. Proceedings of the National Academy of Sciences of the United States of America 112:E1326-E1332.

Etienne, R. S. 2007. A neutral sampling formula for multiple samples and an 'exact' test of neutrality. Ecology Letters 10:608-618.

Etienne, R. S. 2009. Improved estimation of neutral model parameters for multiple samples with different degrees of dispersal limitation. Ecology 90:847-852.

Harpole, W. S., and D. Tilman. 2007. Grassland species loss resulting from reduced niche dimension. Nature 446: 791-793.

Hautier, Y., P. A. Niklaus, and A. Hector. 2009. Competition for light causes plant biodiversity loss after eutrophication. Science 324:636-638.

Hubbell, S. P. 2001. The unified neutral theory of biodiversity and biogeography. Princeton University Press, New Jersey.

Hutchinson, G. E. 1957. Concluding remarks. Cold Spring Harbor Symposia on Quantitative Biology 22:415-427.

Hutchinson, G. E. 1959. Homage to Santa Rosalia or why are there so many kinds of animals? American Naturalist 93:145-159.

Korhonen, J. J., J. Soininen, and H. Hillebrand. 2010. A quantitative analysis of temporal turnover in aquatic species assemblages across ecosystems. Ecology 91:508-517.

Kraft, N. J. B., P. B. Adler, O. Godoy, E. C. James, S. Fuller, and J. M. Levine. 2015. Community assembly, coexistence and the environmental filtering metaphor. Functional Ecology 29:592-599.

Kraft, N. J. B., L. S. Comita, J. M. Chase, N. J. Sanders, N. G. Swenson, T. O. Crist, J. C. Stegen, M. Vellend, B. Boyle, M. J. Anderson, H. V. Cornell, K. F. Davies, A. L. Freestone, B. D. Inouye, S. P. Harrison, and J. A. Myers. 2011. Disentangling the drivers of $\beta$ diversity along latitudinal and elevational gradients. Science 333:1755-1758.

Leibold, M. A., and M. A. McPeek. 2006. Coexistence of the niche and neutral perspectives in community ecology. Ecology 87:1399-1410.

MacArthur, R. H. 1972. Geographical ecology: patterns in the distribution of species. Princeton University Press, New Jersey, US.

MacArthur, R. H., and E. O. Wilson. 1967. The theory of island biogeography. Princeton University Press, New Jersey.

McNaughton, S. J. 1983. Serengeti grassland ecology: the role of composite environmental factors and contingency in community organization. Ecological Monographs 53:291-320.

Menge, B. A., and J. P. Sutherland. 1976. Species diversity gradients: synthesis of the roles of predation, competition, and temporal heterogeneity. American Naturalist 110:351-369.

Mori, A. S., S. Fujii, R. Kitagawa, and D. Koide. 2015. Null model approaches to evaluating the relative role of different assembly processes in shaping ecological communities. Oecologia 178:261-273.

Myers, J. A., J. M. Chase, I. Jiménez, P. M. Jørgensen, A. Araujo-Murakami, N. Paniagua-Zambrana, and R. Seidel. 2013. Beta-diversity in temperate and tropical forests reflects dissimilar mechanisms of community assembly. Ecology Letters 16:151-157.

Paine, R. T. 1966. Food web complexity and species diversity. American Naturalist 100:65-75.

Powell, J. R., S. Karunaratne, C. D. Campbell, H. Yao, L. Robinson, and B. K. Singh. 2015. Deterministic processes vary during community assembly for ecologically dissimilar taxa. Nature Communications 6:8444.

Qian, H., S. Chen, L. Mao, and Z. Ouyang. 2013. Drivers of $\beta$-diversity along latitudinal gradients revisited. Global Ecology and Biogeography 22:659-670.

Sabatini, F. M., B. Jiménez-Alfaro, S. Burrascano, A. Lora, and M. Chytrý. in press. Beta-diversity of Central European 
forests decreases along an elevational gradient due to the variation in local community assembly processes. Ecography doi:10.1111/ecog.02809.

Schmidt, S. K., D. R. Nemergut, J. L. Darcy, and R. Lynch. 2014. Do bacterial and fungal communities assemble differently during primary succession? Molecular Ecology 23:254-258.

Segre, H., R. Ron, N. De Malach, Z. Henkin, M. Mandel, and R. Kadmon. 2014. Competitive exclusion, beta diversity, and deterministic vs. stochastic drivers of community assembly. Ecology Letters 17:1400-1408.

Soininen, J., J. J. Lennon, and H. Hillebrand. 2007. A multivariate analysis of beta diversity across organisms and environments. Ecology 88:2830-2838.

Stachowicz, J. J. 2001. Mutualism, facilitation and the structure of ecological communities. Bioscience 51:235-246.

Stegen, J. C., A. L. Freestone, T. O. Crist, M. J. Anderson, J. M. Chase, L. S. Comita, H. V. Cornell, K. F. Davies, S. P. Harrison, A. H. Hurlbert, B. D. Inouye, N. J. B. Kraft, J. A. Myers, N. J. Sanders, N. G. Swenson, and M. Vellend. 2013a. Stochastic and deterministic drivers of spatial and temporal turnover in breeding bird communities. Global Ecology and Biogeography 22:202-212.

Stegen, J. C., X. Lin, J. K. Fredrickson, X. Chen, D. W. Kennedy, C. J. Murray, M. L. Rockhold, and A. Konopka. 2013 b. Quantifying community assembly processes and identifying features that impose them. The ISME Journal 7:20692079.

Steiner, C. F. 2014. Stochastic sequential dispersal and nutrient enrichment drive beta diversity in space and time. Ecology 95:2603-2612.

Tucker, C. M., L. G. Shoemaker, K. F. Davies, D. R. Nemergut, and B. A. Melbourne. 2016. Differentiating between niche and neutral assembly in metacommunities using null models of $\beta$-diversity. Oikos 125:778-789.

Tuomisto, H. 2010. A diversity of beta diversities: straightening up a concept gone awry. Part 1 . Defining beta diversity as a function of alpha and gamma diversity. Ecography 33:2-22.

Ulrich, W., and N. J. Gotelli. 2010. Null model analysis of species associations using abundance data. Ecology 91 : 3384-3397.

Vellend, M. 2010. Conceptual synthesis in community ecology. The Quarterly Review of Biology 85:183-206.

Vellend, M., D. S. Srivastava, K. M. Anderson, C. D. Brown, J. E. Jankowski, E. J. Kleynhans, N. J. B. Kraft, A. D. Letaw, A. A. M. Macdonald, J. E. Maclean, I. H. Myers-Smith, A. R. Norris, and X. Xue. 2014. Assessing the relative importance of neutral stochasticity in ecological communities. Oikos 123:1420-1430.

Vellend, M., K. Verheyen, K. M. Flinn, H. Jacquemyn, A. Kolb, H. Van Calster, G. Peterken, B. J. Graae, J. Bellemare, O. Honnay, J. Brunet, M. Wulf, F. Gerhardt, and M. Hermy. 2007. Homogenization of forest plant communities and weakening of species-environment relationships via agricultural land use. Journal of Ecology 95:565-573.

Whittaker, R. H. 1960. Vegetation of the siskiyou mountains, Oregon and California. Ecological Monographs 30:279338.

Zhou, J., W. Liu, Y. Deng, Y.-H. Jiang, K. Xue, Z. He, J. D. V. Nostrand, L. Wu, Y. Yang, and A. Wang. 2013. Stochastic assembly leads to alternative communities with distinct functions in a bioreactor microbial community. mBio 4: e00584-12. 